\begin{tabular}{|c|c|c|c|c|c|c|}
\hline \multirow{4}{*}{ Impact Factor: } & ISRA (India) & $=4.971$ & SIS (USA) & $=0.912$ & ICV (Poland) & $=6.630$ \\
\hline & ISI (Dubai, UAE & $=0.829$ & РИНЦ (Russia) & $=0.126$ & PIF (India) & $=1.940$ \\
\hline & GIF (Australia) & $=0.564$ & ESJI (KZ) & $=8.716$ & IBI (India) & $=4.260$ \\
\hline & JIF & $=1.500$ & SJIF (Morocco) & $=5.667$ & OAJI (USA) & $=0.350$ \\
\hline
\end{tabular}

\section{SOI: $1.1 /$ TAS $\quad$ DOI: $10.15863 /$ TAS \\ International Scientific Journal Theoretical \& Applied Science}

p-ISSN: 2308-4944 (print) e-ISSN: 2409-0085 (online)

Year: $2020 \quad$ Issue: 03 Volume: 83

Published: $30.03 .2020 \quad$ http://T-Science.org
QR - Issue

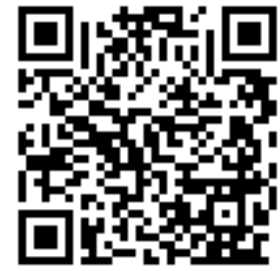

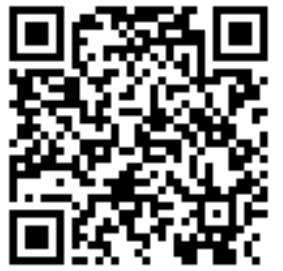

Obid Abdullayev

Samarkand State University

Docent of Department Theoretical and Applied Mechanics

Samarkand, Uzbekistan

abdullaev2006@inbox.ru

\title{
MIXED FINITE ELEMENT METHOD FOR SOLVING ELASTICITY THEORY PROBLEMS
}

Abstract: A mixed finite element method for solving boundary value problems of the theory of elasticity is formulated. The correctness of mixed approximations for stresses (strains) and displacements is investigated. The unique solvability of continuum and finite-dimensional problems is shown.

Key words: elasticity theory, finite element method, mixed scheme, approximation, a priori estimates.

Language: Russian

Citation: Abdullayev, O. (2020). Mixed finite element method for solving elasticity theory problems. ISJ Theoretical \& Applied Science, 03 (83), 145-148.

Soi: http://s-o-i.org/1.1/TAS-03-83-31 Doi: crossef https://dx.doi.org/10.15863/TAS.2020.03.83.31

Scopus ASCC: 2600.

\section{СМЕШАННЫЙ МЕТОД КОНЕЧНЫХ ЭЛЕМЕНТОВ ДЛЯ РЕШЕНИЯ ЗАДАЧ ТЕОРИИ УПРУГОСТИ}

Аннотация: Сформулирован смешанный метод конечных элементов решения краевых задач теории упругости. Исследована корректность смешанных аппроксимаций для напряжений (деформаций) $и$ перемещений. Показана однозначную разрешимость континуальной и конечномерных задач.

Ключевые слова: теория упругости, метод конечных элементов, смешанная схема, аппроксимация, априорные оценки.

Введение

\section{УДК 539.3}

В настоящее время наиболее универсальным методом решения краевых задач теории упругости является метод конечных элементов (МКЭ). Однако, отмечая достоинства классического МКЭ, следует учитывать и его недостатки. К наиболее существенным из них относятся разрывная аппроксимация напряжений и деформаций, а также более низкий порядок сходимости аппроксимации напряжений и деформаций по сравнению с таковым для перемещений. В то же время напряжения обычно являются основными искомыми Функциями в задачах теории упругости, и, следовательно, должны быть определены с достаточно высокой степенью точности.
В связи с этим перспективным в численном анализе задач теории упругости представляется применение смешанных формулировок МКЭ, в которых напряжения и деформации входят в разрешающие уравнения наряду с перемещениями как равноправные неизвестные. Основной выигрыш при использовании смешанных формулировок МКЭ по сравнению с классическим МКЭ в форме метода перемещений состоит в уменьшении погрешности аппроксимации для напряжений и деформаций, а также возможности точного удовлетворения статических граничных условий на поверхности тела. Еще одно преимущество заключается в том, что смешанные схемы МКЭ позволяют обеспечить непрерывность аппроксимации не только для перемещений, но и для напряжений и деформаций. 


\begin{tabular}{|c|c|c|c|c|c|c|}
\hline \multirow{4}{*}{ Impact Factor: } & ISRA (India) & $=4.971$ & SIS (USA) & $=0.912$ & ICV (Poland) & $=6.630$ \\
\hline & ISI (Dubai, UAE & $=0.829$ & РИНЦ (Russia & $=0.126$ & PIF (India) & $=1.940$ \\
\hline & GIF (Australia) & $=0.564$ & ESJI (KZ) & $=8.716$ & IBI (India) & $=4.260$ \\
\hline & JIF & $=1.500$ & SJIF (Morocce & $=5.667$ & OAJI (USA) & $=0.350$ \\
\hline
\end{tabular}

Обобщенная постановка краевой задачи теории упругости. Пусть рассматриваемая тело занимает область $\Omega \subset \mathbb{R}^{n}(n=2,3) \quad$ имеет регулярную границу $Г$. На части границы $\Gamma_{\text {и }}$ заданы перемещения, а на оставшейся части $\Gamma_{6}-$ поверхностные нагрузки. Кроме того, тело подвержено воздействию массовых сил и начальных деформаций.

Вектор - функции, описывающие перемещения точек тела, будим рассматривать как элементы функционального множества:

$U=\left\{V\left|v=\left(v_{i}\right)_{i=1}^{n}, \quad v_{i} \in H^{1}(\Omega), \quad v_{i}\right|_{\Gamma_{u}}=0\right\}(1)$

Множество допустимых тензор-функций для напряжений и деформаций определим формулой:

$$
X=\left\{\tau \mid \tau=\left(\tau_{i j}\right)_{i, j=1}^{n}, \tau_{i j}=\tau_{i j} \in L_{2}(\Omega)\right\} .
$$

Связь между перемещениями и деформациями зададим соотношениями Коши, которые будем записываем в виде:

$$
\varepsilon=B u, \forall u \in U,
$$

где $B$ - линейный дифференциальный оператор, действующий из $U$ в $X$ :

$$
(B V)_{i j}=\frac{1}{2}\left(\frac{\partial V_{i}}{\partial X_{j}}+\frac{\partial V_{j}}{\partial X_{i}}\right) ; i, j=1, . ., n .
$$

Скалярные произведения на пространствах $X$ и $U$ зададим соответствии с формулами:

$$
\begin{aligned}
& (\sigma, \varepsilon)_{x}=\int_{\Omega} \sigma_{i j} \varepsilon_{i j} d \Omega ; \forall \sigma, \varepsilon \in X ; \\
& (u, v)_{U}=(B u, B v)_{x}, \forall u, v \in U .
\end{aligned}
$$

Тогда норма в $U$

$$
\|v\|_{U}: v \in X \rightarrow\|v\|_{U}=(v, v)_{U}^{\frac{1}{2}} .
$$

Норма в $X$

$$
\|\tau\|_{X}: \tau \in X \rightarrow\|\tau\|_{U}=(\tau, \tau)_{U}^{\frac{1}{2}}
$$

Соотношения между напряжениями и деформациями запишем в виде

$$
\sigma=D(\varepsilon-\zeta), \forall \sigma, \varepsilon \in X .
$$

$D$-самосопряженный,

положительно определенный и ограниченный оператор их $X$ в $X$, причем существует $m_{0}$ и $M_{0}$ положительных чисел такие, что

$$
(D \tau, \tau)_{X} \geq m_{0}\|\tau\|_{X}^{2},\|\partial \tau\|_{x} \leq M_{0}\|\tau\|_{X}, \forall \tau \in X .
$$

$U^{*}$ - пространство непрерывных линейных функционалов, определенных на $U$. Значение произвольного линейного функционала $g \in U$ * на элементе $v \in U$ обозначим через $\langle g, v\rangle_{U}$. Тогда норма в $U *$ определяется равенством.

$$
\|g\|_{U *}: g \in U * \rightarrow\|g\|_{U *}=\sup _{v \in U \backslash\{0\}} \frac{|<g, v\rangle_{U} \mid}{\|v\|_{U}} .
$$

Определим на $X \times U$ билинейную форму

$$
\tau, v \rightarrow(\tau, B v)_{X}=\int_{\Omega} \tau_{i j} \frac{1}{2}\left(\frac{\partial v_{i}}{\partial x_{j}}+\frac{\partial v_{j}}{\partial x_{i}}\right) d \Omega .
$$

Тогда в силу вариационного принципа Лагранжа [1] статические соотношения содержатся в уравнении:

$$
(\sigma, B v)_{X}=<\rho, v>_{U}, \forall v \in U, \forall \rho \in U^{*} .
$$

Равенства (3), (9) и (13) позволяют полностью сформулировать краевую задачу теории упругости. Подставляя равенства (3), (9) в (13) получаем:

$$
(D B u, B v)_{X}=\langle\rho, v\rangle_{U}+(D \zeta, B v)_{X}, \forall v \in U .
$$

Уравнение (14) соответствуют классической обобщенной постановке краевой задачи теории упругости в перемещениях. Доказано однозначное разрешимость (14).

Использование уравнения (14) для построения сеточных схем приводит к обычной формулировке МКЭ в форме методы перемещений. В результате, деформации вычисляется не прямо, а путем дифференцирования приближенных значений перемещений, полученных при решении задача в перемещениях, что является основной причиной ухудшения сходимости аппроксимации для напряжений и деформаций по сравнению с таковым для перемещений.

Альтернативный подход состоит в изменении обобщенной постановки краевой задачи таким образом, чтобы напряжения и деформации являлись ее непосредственными аргументами, а не определялись на основе решения задачи в перемещениях. С этой целью представим краевую задачу следующей системой уравнений:

$$
\left\{\begin{array}{l}
(\varepsilon, \kappa)_{X}=(B u, \kappa)_{X}, \forall \kappa \in X \\
(\sigma, \tau)_{X}=(D \varepsilon, \tau)_{X}-(D \zeta, \tau)_{X}, \forall \tau \in X \\
(\sigma, B v)_{X}=<\rho, v>_{U}, \forall v \in U
\end{array}\right.
$$

Таким образом, получаем обобщенную постановку краевой задачи теории упругости относительно перемещений, напряжений и деформаций.

Показано однозначное разрешимость смешанной задачи теории упругости. Получены априорные оценки решений:

$$
\begin{aligned}
\|u\|_{U} & \leq \frac{1}{m_{0}}\|\rho\|_{U^{*}}+\frac{M_{0}}{m_{0}}\|\zeta\|_{X} ; \\
\|\varepsilon\|_{X} & \leq \frac{1}{m_{0}}\|\rho\|_{U^{*}}+\frac{M_{0}}{m_{0}}\|\zeta\|_{X} ; \\
\|\sigma\|_{X} & \leq \frac{M_{0}}{m_{0}}\|\rho\|_{U^{*}}+M_{0}\left(1+\frac{M_{0}}{m_{0}}\right)\|\zeta\| .
\end{aligned}
$$

Построение смешанных проекционносеточных алгоритмов МКЭ основывается на дискретизации исходной континуальной задачи, описываемой системой уравнений (15)-(17). По аналогии определим дискретную задачу 


\begin{tabular}{|c|c|c|c|c|c|c|}
\hline \multirow{4}{*}{ Impact Factor: } & ISRA (India) & $=4.971$ & SIS (USA) & $=0.912$ & ICV (Poland) & $=6.630$ \\
\hline & ISI (Dubai, UAE & $=0.829$ & РИНЦ (Russia) & $=0.126$ & PIF (India) & $=1.940$ \\
\hline & GIF (Australia) & $=0.564$ & ESJI (KZ) & $=8.716$ & IBI (India) & $=4.260$ \\
\hline & JIF & $=1.500$ & SJIF (Morocco) & $=5.667$ & OAJI (USA) & $=0.350$ \\
\hline
\end{tabular}

$$
\begin{aligned}
& \text { следующим образом. Найти тройку } \\
& \left(u_{h}, \sigma_{h}, \varepsilon_{h}\right) \in U_{h} \times X_{h} \times X_{h} \text { такую, что } \\
& \int\left(\varepsilon_{h}, \kappa_{h}\right)_{X}=\left(B u_{h}, \kappa_{h}\right)_{X}, \forall \kappa_{h} \in X_{h} \\
& \left\{\left(\sigma_{h}, \tau_{h}\right)_{X}=\left(D \varepsilon_{h}, \tau_{h}\right)_{X}-\left(D \zeta, \tau_{h}\right)_{X}, \forall \tau_{h} \in X_{h}\right. \\
& \left(\sigma_{h}, B v_{h}\right)_{X}=<\rho, v_{h}>_{U}, \forall v_{h} \in U_{h} \\
& \left\{\begin{array}{l}
\left(R_{h} \hat{\varepsilon}_{h}, R_{h} \hat{\kappa}_{h}\right)_{X}=\left(B u_{h}, \kappa_{h}\right)_{X} \forall \kappa_{h} \in X_{h} \\
\left(R_{h} \hat{\sigma}_{h}, R_{h} \hat{\tau}_{h}\right)_{X}=\left(D R_{h} \hat{\varepsilon}_{h}, \hat{\tau}_{h}\right)_{X}-\left(D \zeta, R_{h} \hat{\tau}_{h}\right)_{X}, \forall \hat{\tau}_{h} \in Z_{h} \\
\left(R_{h} \hat{\sigma}_{h}, B \hat{v}_{h}\right)_{X}=<\rho, P_{h} \hat{v}_{h}>_{U}, \forall \hat{v}_{h} \in V_{h}
\end{array}\right.
\end{aligned}
$$

где $U_{h} \subset U, X_{h} \subset X \quad$ - $\quad$ конечномерные подпространства для перемещений и напряжений (деформаций). Система уравнений (21) - (23) можно представить в виде:

$V_{h}, Z_{h}$ - конечномерные пространства узловых перемещений, и деформаций $P_{h}, R_{h}$ - операторы продолжения из $V_{h}$ в $U_{h}$, из $Z_{h}$ в $X_{h} . B_{h}=B P_{h}$ линейный оператор, действующей из $V_{h}$ на $Y_{h}$ где $Y_{h}$ образ оператора $B_{h}$.

Обозначим через $P_{h}^{*}, R_{h}^{*}, B_{h}^{*}$ - операторы сопряженные к $P_{h}, R_{h}, B_{h}$ соответственно.

Оператор $M_{h}$ отображающий $Z_{h}$ в $Z_{h}^{*}$ определим следующим образом:

$$
M_{h}=R_{h}^{*} R \text { в } Z_{h}^{*} .
$$

Оператор $G_{h}$ отображающий $Z_{h}$ на $Z_{h}^{*}$ определим следующим образом

$$
G_{h}=R_{h}^{*} D P_{h} \text { в } Z_{h}^{*} .
$$

Оператор $H_{h}$ отображающий $V_{h}$ на $Z_{h}^{*}$ определим следующим образом

$$
H_{h}=R_{h}^{*} B_{h} \text { в } Z_{h}^{*} \text {. }
$$

Тогда $H_{h}^{\prime}$ транспозиция оператора $H_{h}$, отображает $Z_{h}$ на $V_{h}^{*}$ и определяется следующим образом

$$
H_{h}^{\prime}=B_{h}^{*} P_{h} \text { в } V_{h}^{*} .
$$

Систему уравнений (24)-(26) можно представить в виде

$$
\left\{\begin{array}{l}
M_{h} \hat{\varepsilon}_{h}=H_{h} \hat{u}_{h} \text { в } Z_{h}^{*} \\
M_{h} \hat{\sigma}_{h}=G_{h} \hat{\varepsilon}_{h}-\hat{\xi}_{h}, \text { в } Z_{h}^{*} \\
H \hat{\sigma}_{h}=\hat{\rho}_{h}
\end{array}\right.
$$

где $\xi_{h}=R_{h}^{*} D \zeta \in Z_{h}^{*} u \hat{\rho}_{h}=P_{h}^{*} \rho \in V_{h}^{*}$.

Если представить $G_{h}$ в виде: $G_{h}=M_{h} \hat{D}_{h}$ в $Z_{h}^{*}$, тогда систему уравнений (31) можно представить в виде:

$$
\left\{\begin{array}{c}
M_{h} \hat{\varepsilon}_{h}=H_{h} \hat{u}_{h}, \mathrm{~B} Z_{h}^{*} \\
\hat{\sigma}_{h}=\hat{D}_{h}\left(\hat{\varepsilon}_{h}-\hat{\zeta}_{h}\right), \mathrm{B} Z_{h} \\
H_{h}^{\prime} \hat{\sigma}_{h}=\hat{\rho}_{h}, \mathrm{~B} V_{h}
\end{array}\right.
$$

Уравнения (32)-(34) могут быть представлены в форме одного операторного уравнения относительно перемещений, т.е.

$$
A_{h} \hat{u}_{h}=\hat{f}_{h} \text { в } V_{h}^{*} .
$$

В котором линейный оператор $A_{h}: V_{h} \rightarrow V_{h}^{*}$ определяется выражением

$$
A_{h}=H_{h}^{\prime} \hat{D}_{h} M_{h}^{-1} H_{h} \text { в } V_{h}^{*} .
$$

А элемент $\hat{f}_{h}=V_{h}^{*}$ имеем вид:

$$
\hat{f}_{h}=\hat{\rho}_{h}+H_{h}^{\prime} \hat{D}_{h} \hat{\zeta}_{h} \in V_{h}^{*} \text {. }
$$

Показано, что решения уравнения (35) существует, единственно и непрерывно зависит от правой части, т.е. от элемента $\hat{f}_{h} \in V_{h}^{*}$. При этом имеет место оценки

$$
\begin{aligned}
\left\|u_{h}\right\|_{U} & \leq \frac{1}{\delta_{0} d_{0}}\left\|\hat{f}_{h}\right\|_{V_{h}^{*}} \\
\left\|\varepsilon_{h}\right\|_{X} & \leq \frac{1}{\delta_{0}}\left\|\hat{f}_{h}\right\|_{V_{h}^{*}} \\
\left\|\sigma_{h}\right\|_{X} & \leq \frac{\Delta_{0}}{\delta_{0}}\left\|\hat{f}_{h}\right\|_{V_{h}^{*}}+\Delta_{0}\left\|\hat{\zeta}_{h}\right\|_{Z_{h}} .
\end{aligned}
$$

Учитывая (37) априорные оценки (38)-(40) примут вид:

$$
\begin{aligned}
\left\|\hat{u}_{h}\right\|_{V_{h}} & \leq \frac{1}{\delta_{0}}\left\|\hat{\rho}_{h}\right\|_{V_{h}^{*}}+\frac{\Delta_{0}}{\delta_{0}}\left\|\hat{\zeta}_{h}\right\|_{Z_{h}} ; \\
\left\|\hat{\varepsilon}_{h}\right\|_{Z_{h}} & \leq \frac{1}{\delta_{0}}\left\|\hat{\rho}_{h}\right\|_{V_{h}^{*}}+\frac{\Delta_{0}}{\delta_{0}}\left\|\hat{\zeta}_{h}\right\|_{Z_{h}} ; \\
\left\|\hat{\sigma}_{h}\right\|_{Z_{h}} & \leq \frac{\Delta_{0}}{\delta_{0}}\left\|\hat{\rho}_{h}\right\|_{V_{h}^{*}}+\Delta_{0}\left(1+\frac{\Delta_{0}}{\delta_{0}}\right)\left\|\hat{\zeta}_{h}\right\|_{Z_{h}} .
\end{aligned}
$$

Предложенный итерационный процесс сходится со скоростью геометрической прогрессии независимо от выбора начального приближения [6-10] 


\begin{tabular}{|c|c|c|c|c|c|c|}
\hline \multirow{4}{*}{ Impact Factor: } & ISRA (India) & $=4.971$ & SIS (USA) & $=0.912$ & ICV (Poland) & $=6.630$ \\
\hline & ISI (Dubai, UAE & $=0.829$ & РИНЦ (Russia & $=0.126$ & PIF (India) & $=1.940$ \\
\hline & GIF (Australia) & $=0.564$ & ESJI (KZ) & $=8.716$ & IBI (India) & $=4.260$ \\
\hline & JIF & $=1.500$ & SJIF (Morocce & $=5.667$ & OAJI (USA) & $=0.350$ \\
\hline
\end{tabular}

\section{References:}

1. Voroshko, P.P. (1985). Smeshanniye variasionniye formulirovki zadach teorii uprugosti i ix realizasiya metodom konechnix elementov. Problema prochnosti, №1, pp.100105.

2. Zenkevich, O. (1975). Metod konechnix elementov v texnike. (p.560). Moscow: Mir.

3. Marchuk, G.I., \& Agoshkov, V.I. (1981). Vvedeniye $v$ proyeksionno-setochniye metodi. (p.416). Moscow: Nauka.

4. Chirkov, A.Yu. (2003). Primeneniye smeshannoy approksimasii $\mathrm{k}$ resheniyu dvumernix zadach teorii uprugosti metodom konechnix elementov. Problemi prochnosti, №6.

5. Lavigin, D.S. (2019). Smeshanniy metod konechnix elementov $\mathrm{v}$ trexmernix zadachax teorii uprugosti. Sovremenniye problemi nauki $i$ obrazovaniya, №5.
6. Marchuk, G.I., \& Agoshkov, V.I. (1981). Vvedeniye $v$ proyeksionno-setochniye metodi. (p.416). Moscow: Nauka.

7. Morozkin, N.D., Abdullayev, O., Nugumanov, E.R., Axmetshina, G.A., \& Kolonskix, D.M. (2008). Smeshannaya proyeksionno-setochnaya sxema dlya resheniya zadach teorii uprugosti. Vestnik BashGU, №1, pp.4-8.

8. Saad, Y. (2003). Iterative Methods for Spores Linear System. (p.546). STAN.

9. Uotkins, D.S. (2006). Osnovi matrichnix vichisleniy. (p.672). Moscow: BINOM. Laboratoriya znaniy.

10. Chirkov, A.Yu. (2005). Primeneniye v konechno-elementnix raschetax modifisirovannogo algoritma metoda sopryajennix gradiyentov. Problem prochnosti, №6, Kiyev, pp. 89-102. 